index ecomunicación | no 11(2) 2021 | Páginas 187-208

E-ISSN: 2174-1859 | ISSN: 2444-3239 | Depósito Legal: M-19965-2015

Recibido el 24_03_2021 | Aceptado el 08_05_2021 | Publicado el 15_07_2021

\title{
MAPEO DEL CONSUMO DE MEDIOS EN LOS JÓVENES: REDES SOCIALES, 'FAKE NEWS' Y CONFIANZA EN TIEMPOS DE PANDEMIA
}

\section{MAPPING MEDIA CONSUMPTION AMONG YOUNGEST: SOCIAL NETWORKS, FAKE NEWS AND TRUSTWORTHY IN PANDEMIC TIMES}

https://doi.org/10.33732/ixc/11/02Mapeod

\begin{abstract}
Ana Pérez-Escoda Universidad Antonio de Nebrija de Madrid aperezes@nebrija.es https://orcid.org/0000-0002-4895-0043
\end{abstract}

Gema Barón-Dulce Universidad Antonio de Nebrija de Madrid gbaron@nebrija.es https://orcid.org/0000-0002-1938-1224

Juana Rubio-Romero Universidad Antonio de Nebrija de Madrid jrubio@nebrija.es https://orcid.org/0000-0002-9389-6269

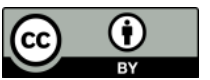

Para citar este trabajo: Pérez-Escoda, A., Barón-Dulce, G. y RubioRomero, J. (2021). Mapeo del consumo de medios en los jóvenes: redes sociales, 'fakes news' y confianza en tiempos de pandemia. index.comunicación, 11(2), 187-208. https://doi.org/10.33732/ixc/11/02Mapeod 
Resumen: La explosión de la pandemia por Covid-19 ha supuesto una transformación importante en el consumo de medios y el uso de redes sociales. Nuevos hábitos y exposiciones prolongadas a dispositivos conectados sumados a cantidades ingobernables de información alertan de una realidad preocupante, especialmente entre la población joven. El objetivo de esta investigación es descubrir el grado de confianza de la generación $\mathrm{Z}$ hacia los medios, sus preferencias de consumo en redes y la asociación que realizan entre consumo de medios y fake news. Utilizando una metodología cuantitativa de carácter descriptivo y exploratorio, se presenta un estudio con una muestra de 225 jóvenes pertenecientes a este nicho poblacional. El estudio aborda tres dimensiones: consumo de medios, de redes sociales y percepción frente a las fake news. Los resultados apuntan a que esta generación consume intensivamente los medios en los que menos confianza deposita y confía en los medios que menos consume. Las conclusiones indican que las redes constituyen la principal fuente de consumo de información de esta generación, entre otros contenidos, pese a ser según sus percepciones la fuente menos fiable y la más propensa a distribuir fake news. Se denota una falta de alfabetización mediática desde una perspectiva más crítica que formativa.

Palabras clave: Redes sociales; consumo de medios; fake news; generación $\mathrm{Z}$, pandemia.

Abstract: The explosion of the Covid-19 pandemic has led to a major transformation in media consumption and the use of social networks. New habits and extensive exposure to connected devices coupled with unmanageable amounts of information warn of a worrying reality, especially among the younger population. The aim of this research is to discover the degree of trustworthiness of Generation $\mathrm{Z}$ towards the media, their media consumption preferences and the association they make between media consumption and fake news. Using a descriptive and exploratory quantitative methodology, a study is presented with a sample of 225 young people belonging to this population niche. The study addresses three dimensions: media consumption, social networks and perception of fake news. The results show that generation $\mathrm{Z}$ is an intensive consumer of the media they trust the least and perceive traditional media as the most trustworthy. The findings indicate that social networks are the main source of information consumption for this generation, among other content, despite also being the least trustworthy and the most likely to distribute fake news according to their perceptions. There is a lack of media literacy from a critical rather than a formative perspective.

Keywords: Social Networks; Media Consumption; Fake News; Generation Z, Pandemic. 


\section{Introducción}

Tal y como arroja el último informe We Are Social (2021), cada vez son más escasos los españoles reacios a utilizar las redes sociales. Muy al contrario, durante 2020, el año de la pandemia, España sumó más de 8 millones de nuevos usuarios de redes sociales, si bien estas siguen estando un 1,1\% por debajo en tiempo de uso frente a las apps de mensajería. El mismo informe revela que las redes más populares en España son YouTube, que sigue liderando el listado con más de 37 millones de usuarios potenciales (89,3\%), seguido de Facebook (79,2\%), Instagram (69\%) y Twitter (52,6\%). La red que más crece es Pinterest, que suma un millón de nuevos usuarios.

Pero si hay algo que ha revelado este estudio es la creciente preocupación de los españoles por no saber reconocer las fake news (65,1\%). En este sentido, algunos autores (Rodríguez-Fernández, 2019; Valero y Oliveira, 2018) señalan que la acepción fake news ha sido elegida palabra del año en diccionarios británicos como Collins y Oxford, que la definen como información falsa, a menudo sensacionalista, diseminada bajo la apariencia de noticia. En la misma línea, Blanco Alfonso et al. (2019) coinciden en que son contenidos producidos con apariencia de noticias, pero matizan que ni se procesan ni se distribuyen por los cauces habituales del periodismo. En cambio, en el discurso contemporáneo, especialmente en el de los medios de comunicación, se interpretan como publicaciones virales con contenidos ficticios procedentes también de cuentas ficticias (Tandoc et al., 2018). A esto se une la percepción de la población sobre lo que es verdad y lo que no, como recientemente han señalado Tayal y Bharathi (2021).

En cualquier caso, la proliferación de este tipo de noticias es un problema que afecta a toda la ciudadanía, pero, particularmente, a los jóvenes españoles, que tienden a confiar en las redes sociales para mantenerse informados (Mendiguren, Pérez-Dasilva y Meso-Ayerdi, 2020). Según los mismos autores, se extrae la conclusión de que los usuarios más jóvenes valoran el hecho de que las noticias estén continuamente disponibles para mantenerse informados sobre las historias más relevantes o curiosas y poder compartirlas, en la línea de lo que sugieren otros autores que, basándose en estudios impulsados por distintas universidades españolas (Negredo et al., 2020; Yuste, 2020), concluyen que los jóvenes estudiantes no tienen interés por estar informados a través de los medios de comunicación convencionales, como el periódico o la radio, pues priorizan los medios audiovisuales de consumo inmediato.

Este comportamiento es coherente con otras características significativas de la llamada generación Z, definida como los niños y adolescentes que nacieron entre 1995 y 2012 (Schroer, 2008) y que tienen en 
común el uso nativo de las Tecnologías de la Información y la Comunicación (TIC), la interacción, la rapidez, la impaciencia y la resiliencia, entre otros, como bien ha sido descrito por Herrero-Diz, Conde-Jiménez y Reyes de Cózar (2020) y Sádaba y Pérez-Escoda (2020).

Esta preferencia, basada en la inmediatez, genera también un problema de desinformación. La Comisión Europea (2018) definió la palabra desinformación como «información falsa, imprecisa, o engañosa, presentada y promovida para obtener ingresos o causar daño público intencionadamente». El estudio de Yuste (2020) revela que la desinformación ha sido uno de los temas más controvertidos durante esta crisis y uno de los retos que deben ser abordados teniendo en cuenta las nuevas formas de consumir información de los jóvenes.

Un ejemplo de aplicación de consumo inmediato que es la más utilizada por la generación Z junto con YouTube, (IAB, 2020) es la aplicación de mensajería instantánea WhatsApp. Esta aplicación se une a las demás redes sociales como una de las preferidas por los jóvenes para informarse (Newman et al., 2017). En este contexto hiperconectado, las noticias se difunden a una gran velocidad, dándose por verdaderas, sin contrastarse, siquiera, $\mathrm{y}$, como concluye el estudio realizado por Mendiguren et al. (2020), un 87\% de los jóvenes reconoce haber dado por verídica una información que no lo era. Así, no sorprende que los jóvenes se muestren preocupados por identificar la veracidad o no de las noticias, pero también preocupa el hecho de que ellos mismos consideran tener más criterio que sus padres para identificar las fake news por su involucración como generación digital en el mundo de las tecnologías y las competencias digitales autopercibidas (Pérez-Escoda, LenaAcebo y García-Ruiz, 2021).

\subsection{Las redes sociales como agentes de cambio}

Desde su llegada y generalización en la primera década de los 2000, ha existido siempre algo de confusión a la hora de identificar los medios y las redes sociales. Si bien en inglés, lengua original de este concepto, la denominación más extendida es social media, en español se utiliza más habitualmente el término 'redes sociales' para denominar a los medios sociales, que son aquellas herramientas tecnológicas para comunicarnos o explotar aquellos nodos o individuos de nuestras redes sociales», siendo entonces las redes sociales los nodos o lazos que nos unen (Barón-Dulce, 2017).

Desde su implantación en el panorama de medios de los españoles, en torno a 2010, el uso de las redes sociales y su extensión no ha dejado de crecer y modificar el panorama comunicativo (Pérez-Tornero, 2020; Pérez-Escoda et 
al., 2021). Tal y como se indica en la introducción de este artículo, en el último informe de We Are Social (2021), las redes sociales más utilizadas en España son WhatsApp (89,5\%), YouTube $(89,3 \%)$ y Facebook $(79,2 \%)$. De cerca les sigue Instagram, con un 69\% de los encuestados de entre 16 y 64 años.

Si nos centramos en el uso de las redes sociales por parte de los jóvenes miembros de la generación Z, vemos que prefieren utilizar Spotify, TikTok, Snapchat, Twitch, Tumblr, 21Buttons, HouseParty y Peoople frente a Facebook, Linkedin, Twitter y Telegram, que es lo que más utilizan los jóvenes milénicos. WhatsApp, YouTube, Instagram y Pinterest son las más transversales entre ambos grupos generacionales (IAB, 2020). Ambas son las generaciones que más tiempo pasan conectadas a las redes sociales.

Analizando el panorama informativo, vemos un cambio de tendencia puesto que la generación $\mathrm{Z}$ ha cambiado la información de la televisión por la información que le ofrecen las redes sociales (Espiritusanto, 2016; Patch, 2018). El mismo autor señala que el fenómeno llamado 'periodismo ciudadano' por el que los medios de comunicación tradicionales han ido perdiendo credibilidad en este grupo generacional, se suma a la proclamada muerte del papel.

\begin{abstract}
Los periódicos comienzan a verse como un producto que solo vende las noticias de ayer, a las que el público ya ha accedido a través de la webs y redes sociales. La información está en otros lugares y se produce también por otros canales y en formatos y lenguajes distintos. La generación $Z$ se forma e informa en tiempo real, entre todos escriben esos borradores de la historia, han nacido haciéndolo y no ven una autoridad moral o superior en los periodistas o los medios de comunicación tradicionales para que lo hagan por ellos. No son una audiencia pasiva, sino que complementan su información con el New York Times o el «youtuber» de turno. Ambos en un mismo rango de autoridad informativa, el que ellos deciden darle (Espiritusanto, 2016:121).
\end{abstract}

En este contexto, sin duda, los medios sociales parecen sustituir a los medios de comunicación tradicionales cada vez en más segmentos poblacionales, lo que supone un hándicap para los medios tradicionales que no encuentran entre sus audiencias a los segmentos más jóvenes (Gómez Calderón et al., 2020; Levy et al., 2019; Pedrero-Esteban, 2014; Pérez-Maíllo et al., 2018). Si bien en el informe Digital News Report 2019, hasta un 38\% de los españoles del tramo de 18 a 24 años prefería las redes sociales y blogs para obtener información (un 40\% en el estudio de 2020), con la llegada de la crisis del Coronavirus y el aumento de los bulos derivado del momento de crispación que vivimos, la edición de 2020 del mismo estudio identifica un descenso de la confianza de los ciudadanos en los medios de comunicación, alcanzando la confianza en las noticias en general un 36\%, la cifra más baja en 5 años. También se extrae que los ciudadanos culpan al gobierno, los partidos políticos 
y las redes sociales del aumento de las noticias falsas en un 63\% (Negredo et al., 2020). En este estudio, se aprecia también que España es un país extraordinariamente participativo donde se empieza «a compartir menos y comentar más» y los jóvenes eligen para ello Facebook (44\%) y WhatsApp (34\%), junto con YouTube (27\%). Aun así, se aprecia un fuerte crecimiento de Twitter (26\%) y de Instagram (18\%), fundamentalmente en mujeres.

\subsection{La generación $Z$ y el consumo de medios}

Educados en plena crisis, los jóvenes que se pueden clasificar en la generación Z, también denominados centenials son casi el 26\% de la población y más de ocho millones de los habitantes de España (Quid, 2017). Según este mismo estudio, es una generación que, a priori, no cree en las marcas y contrasta todos sus mensajes. En la misma línea, el informe de 2021 de la consultora de investigación Quantilope (2021) subraya que los centenials «quieren ver por su parte algo verdaderamente auténtico y consistente» por parte de las marcas.

Esta generación, con su particular idiosincrasia, tiene unos rasgos comunes y la mayor parte de los autores coinciden en realzar el interés que tienen y el uso intensivo que realizan de las tecnologías de la información y la comunicación, el hábito de comunicarse, relacionarse, generar y compartir contenido y buscar y hallar lo que demandan en tiempo real (Álvarez-Ramos, Heredia-Ponce y Romero, 2019; Patch, 2018; Schroeder, 2008; Rubio-Romero y Barón -Dulce, 2019). Asimismo, Carrera, Blanco-Ruiz y Sainz (2020) afirman que:

La confluencia de Internet y el auge de los teléfonos inteligentes (Smartphone) están siendo de tal calado que han transformado el mundo tal y como lo conocíamos, influyendo por tanto en la identidad de las nuevas generaciones. La forma en la que la «Gen Z» se relaciona, se forma e informa no puede entenderse sin la irrupción y combinación de estas tecnologías (p.568).

En este sentido, y siguiendo la relación de los jóvenes y la comunicación, Álvarez-Ramos, Heredia-Ponce y Romero (2018: 73) destacan que, para esta generación, «la interacción entre comunicación, tecnología y sociedad ha supuesto una verdadera revolución que está condicionando la manera en la que se construyen y distribuyen los discursos en el nuevo milenio». En el estudio realizado por Carrera et al. (2020) hallaron que los resultados sobre el consumo mediático de la juventud española «reflejan una adolescencia transmedia integrada por usuarios-fans que transitan entre distintas modalidades de relato mainstream, de las novelas a las películas, de los canales de YouTube a los libros autobiográficos, de las películas a Instagram, etc.». Las autoras destacan en su informe que Internet se ha convertido en un medio de referencia para el ocio y el 
entretenimiento, pero el componente ideológico que subyace a los procesos comunicativos en Internet deja ver a los prosumers que hay en ellos.

\subsection{Las fake news y la infodemia, males endémicos postpandemia}

El término 'infodemia', referido a la sobreabundancia de información, ya sea rigurosa o falsa, no es un término nuevo en sentido estricto, sin embargo, con la pandemia por Covid-19 se ha dimensionado de forma inesperada (OMS, 2020) y alude a la dificultad que supone encontrar fuentes y orientación fiable debido al exceso de información. Ya antes de la pandemia COVID 19, el Foro Económico Mundial advertía que la infodemia es uno de los diez riesgos globales del futuro, y que el $51 \%$ de los expertos considera que esta situación no mejorará en los próximos años y en 2022 la mitad de las noticias serán fake news (Herrero Diz, Conde-Jiménez y Reyes de Cózar, 2020).

El «I Estudio sobre el Impacto de las Fake News en España», llevado a cabo en 2017 por la empresa de estudios de mercado Simple Lógica y el grupo de investigación en Psicología del Testimonio de la Universidad Complutense de Madrid (UCM), ya revelaba que el 86\% de los españoles tenía dificultades para distinguir entre 'fake news' y 'noticias verdaderas', aduciendo como principales motivos para saber distinguir una noticia falsa: la irrealidad del contenido (29,6\%); el medio en el que aparece publicada (26,9\%); los titulares demasiado alarmistas, ridículos o improbables (17,9\%); el autor de la noticia $(7,7 \%)$; contraste o verificación de la información (5,8\%); y sentido común, lógica y cultura $(3,1 \%)$. La misma investigación revelaba que el $78 \%$ de los encuestados entre 16 y 24 años no ha sabido diferenciar una noticia falsa de una verdadera, porcentaje que se eleva al $92 \%$ en el caso de los participantes mayores de 55 años.

En el contexto europeo, desde 2015 se trabaja para luchar contra la desinformación ante la preocupación que supone la proliferación de fake news y la amenaza a las sociedades democráticas. El Eurobarómetro de otoño 2019 (Comisión Europea, 2019) refleja una mayor preocupación de los españoles (86\%) frente a los europeos (74\%). También este mismo Eurobarómetro señala que la desconfianza hacia las redes sociales es mayor en España (73\%) que en el resto de países europeos (65\%). En este contexto de sobreabundancia informativa y descentralización de la información es donde las fake news tienen su caldo de cultivo. Entendemos por fake news la información errónea que, bajo la apariencia de noticia periodística, se difunde deliberadamente para promover intereses particulares y que induce a error a los lectores (Allcot \& Gentzkow, 2017).

Los principales focos de producción de noticias falsas son las redes sociales y las páginas webs. Según Gómez Calderón et al. (2020), a través de las 
redes sociales su difusión es más extensa, rápida y profunda que las informaciones veraces, debido a las reacciones que suscitan y la rapidez de alcance con el público. El Digital News Report.es 2020 (Negredo et al., 2020) recoge una serie de noticias que dan cuenta de la preocupación en nuestro país por los bulos: "El 65\% de los encuestados españoles afirma que le preocupa no saber discernir lo que es verdadero o falso en internet", un porcentaje ligeramente inferior a años pasados, aunque sigue ocupando un lugar principal frente a la media europea (56\%); el porcentaje español es algo inferior a Portugal (76\%) y está lejos de los países con menor polarización política, como Holanda (32\%) o Alemania (37\%). Según dicho estudio, la población más preocupada son los mayores de 55 años y son los jóvenes entre 18-24 años (generación Z) los menos preocupados (58\%), lo que nos hace centrar la atención de estudio en este nicho poblacional.

\subsection{Objetivos y preguntas de investigación}

El contexto descrito en los epígrafes anteriores nos da cuenta de un panorama que demanda un análisis descriptivo y exploratorio con el objetivo principal de descubrir el grado de confiabilidad entre la generación $\mathrm{Z}$ hacia los medios, sus preferencias de consumo en redes y la asociación que realizan entre consumo de medios y fake news. Sólo este tipo de estudios pueden ayudar a instituciones y políticos a diseñar estrategias formativas que combatan la 'pandemia informativa' que nuestra sociedad sufre.

Para este propósito se establecieron las siguientes preguntas de investigación (P.I.):

1. ¿Qué medios consume la generación Z?

2. ¿Qué grado de confiabilidad les otorgan?

3. ¿Qué redes sociales consumen en mayor medida?

4. ¿Qué rol adoptan en su interacción en redes?

5. ¿Son capaces de distinguir fake news y qué redes sociales asocian más a la distribución de fake news?

\section{Metodología}

La metodología elegida para la investigación fue cuantitativa, de carácter descriptivo y exploratorio, que es la adecuada en este tipo de estudios (Creswell y Poth, 2016). Para la recogida de datos se optó por el cuestionario como herramienta adecuada con un diseño ad hoc que analizara las variables de estudio necesarias (Vilches, 2012). La definición de las variables de estudio se vertebró a partir de tres constructos de investigación cuyos resultados 
sirvieron para dar respuesta a las preguntas de investigación: 1) Consumo de medios (P.I. 1 y 2), 2) consumo de redes sociales (P.I. 3 y 4) y 3) desinformación y fake news (P.I. 5).

La selección de la muestra fue intencional y de conveniencia, siendo los estudiantes pertenecientes a tres universidades (Universidad Nebrija, Universidad de Cantabria y Universidad Politécnica de Valencia) procedentes de provincias distintas, pero todos ellos con la característica común de pertenecer a la generación Z. La muestra final estuvo compuesta por 225 sujetos pertenecientes a titulaciones de Ciencias Sociales (Comunicación, Educación, Marketing y Audiovisual). La recogida de datos se realizó entre octubre de 2020 y enero de 2021 coincidiendo con la tercera ola de la pandemia. Todos los sujetos de estudio estaban en el rango etario 18-22 $(M=19,52 ;$ D.T.=0.907; Min.=18; Máx.=22) (figura 1).

Tabla 1. Descripción de la muestra por edad, género y provincia de procedencia

\begin{tabular}{|c|c|c|c|}
\hline \multicolumn{2}{|r|}{ Variable } & Frecuencia & Porcentaje \\
\hline \multirow{5}{*}{ Edad } & 18 & 24 & 10,7 \\
\hline & 19 & 92 & 40,9 \\
\hline & 20 & 87 & 38,7 \\
\hline & 21 & 13 & 5,8 \\
\hline & 22 & 9 & 4 \\
\hline \multirow{2}{*}{ Género } & Femenino & 149 & 66,2 \\
\hline & Masculino & 76 & 33,8 \\
\hline \multirow{13}{*}{ Provincia } & Guipúzcoa & 2 & 0,9 \\
\hline & Andalucía & 3 & 1,3 \\
\hline & Aragón & 1 & 0,4 \\
\hline & Asturias & 2 & 0,9 \\
\hline & Canarias & 7 & 3,1 \\
\hline & Cantabria & 29 & 12,9 \\
\hline & Castilla La Mancha & 7 & 3,1 \\
\hline & Castilla y León & 11 & 4,9 \\
\hline & Comunidad Valenciana & 56 & 24,9 \\
\hline & Extremadura & 1 & 0,4 \\
\hline & Galicia & 7 & 3,1 \\
\hline & Islas Baleares & 2 & 0,9 \\
\hline & Madrid & 97 & 43,1 \\
\hline
\end{tabular}

Fuente: elaboración propia. 
Figura 1. Gráfico de cajas para la distribución de la muestra según edad y género

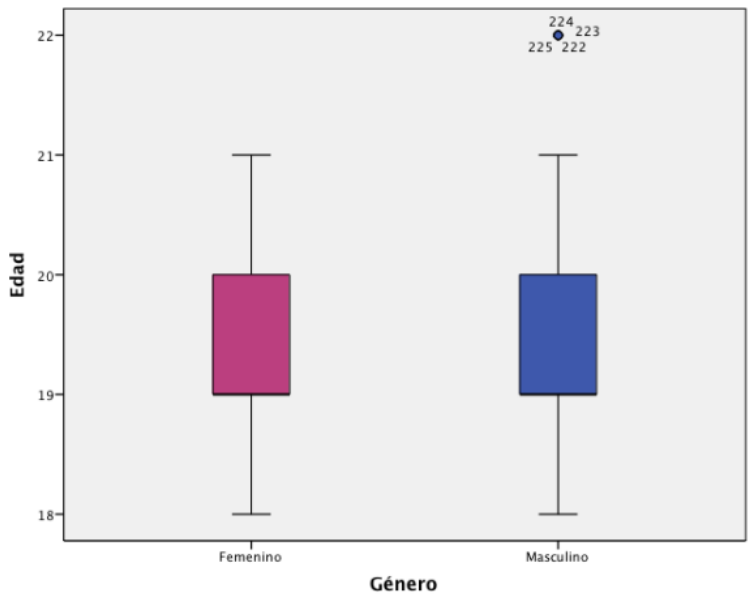

Fuente: elaboración propia.

El cuestionario final fue el resultado de un doble proceso: 1) primero el equipo diseñó un cuestionario en el que se establecieron cuatro bloques diferenciados: a) variables sociodemográficas; b) variables relacionadas con el consumo de medios; c) variables relacionadas con el consumo de redes sociales y d) variables relacionadas con la desinformación y las fake news. Una vez definidos los bloques, el cuestionario se envió a un panel de expertos para su validación $(n=5)$ tratando de buscar coherencia interna y claridad para los constructos de estudio. Tras esta fase en la que se eliminaron siete ítems y se redactaron nuevamente ocho, el cuestionario quedó configurado por 100 ítems organizados en los constructos mencionados. La herramienta de distribución del cuestionario fue GoogleForms y se recogió consentimiento de cada individuo antes de realizar libremente la encuesta.

El análisis estadístico empleado en las encuestas fue descriptivo, basado en frecuencias, cuyas respuestas se analizaron en porcentajes como se muestra en el apartado de los resultados. Se utilizó para ello el paquete estadístico SPSS versión 24. Antes de dicho análisis se procedió a analizar la fiabilidad estadística del instrumento a partir de los valores obtenidos en el alfa de Crombach resultante por bloques o dimensiones estudiadas. El índice de consistencia interna dio resultados superiores a 0.7, por lo que puede considerarse aceptable la consistencia del instrumento, haciéndolo válido para el estudio planteado (González y Pazmiño, 2015). 


\section{Resultados}

Los resultados que aquí se presentan son una muestra parcial de la investigación en su primera etapa. La presentación de los resultados se hace a partir de las respuestas de investigación planteadas y de los tres bloques de estudio: 1) Consumo de medios, 2) Consumo de redes y 3) Distinción de fake news.

\subsection{Consumo de medios y fiabilidad otorgada}

En este bloque los resultados dan respuesta a la primera pregunta de investigación: ¿Qué medios consume la generación $\mathrm{Z}$ y qué grado de confiabilidad le otorga? Para su respuesta se utilizaron 16 ítems específicos repartidos en ocho para cada una de las dos variables estudiadas: $V_{1}=$ Fuentes a través de la cual te informas habitualmente (8 ítems) y $V_{2}=$ Grado de fiabilidad que otorgas a esas fuentes (8 ítems). Ambas variables de estudio eran cualitativas, categóricas y ordinales con respuesta tipo Likert donde 1=Nunca, $2=$ Poco, $3=$ Ocasionalmente, $4=$ Con frecuencia y $5=$ Siempre.

Así para la primera variable se presenta un estudio de la media, desviación típica y frecuencias en porcentajes como se observa en la tabla 2.

Tabla 2. Análisis de la media, la desviación típica $y$ la frecuencia para el estudio de la $V_{1}$

\begin{tabular}{|l|r|r|r|r|r|r|r|r|}
\hline $\begin{array}{c}\text { V1: } \\
\text { Habitualmente } \\
\text { te informas a } \\
\text { través de ... }\end{array}$ & Media & \multicolumn{1}{|c|}{ D.T. } & $\begin{array}{c}\text { Nunca } \\
\mathbf{( \% )}\end{array}$ & $\begin{array}{c}\text { Poco } \\
(\%)\end{array}$ & $\begin{array}{c}\text { Ocasion } \\
\text { almente } \\
(\%)\end{array}$ & $\begin{array}{c}\text { Con } \\
\text { frecuenc } \\
\text { ia (\%) }\end{array}$ & $\begin{array}{c}\text { Siempr } \\
\text { e (\%) }\end{array}$ & N \\
\hline Radio receptor & 1,71 & 0,96 & $\mathbf{5 3 , 3}$ & 32,4 & 6,2 & 6,2 & 1,8 & 225 \\
\hline Radio online & 1,61 & 0,875 & $\mathbf{5 9 , 1}$ & 26,7 & 8,9 & 4,9 & 0,4 & 225 \\
\hline Periódicos & 1,7 & 0,884 & $\mathbf{5 2 , 4}$ & 30,2 & 12,4 & 4,4 & 0,4 & 225 \\
\hline Prensa digital & 3,33 & 1,085 & 6,7 & 12,9 & 36 & 29,8 & 14,7 & 225 \\
\hline Televisión & 3,45 & 1,161 & 5,8 & 16,4 & 25,3 & $\mathbf{3 1 , 6}$ & 20,9 & 225 \\
\hline TV online & 2,23 & 1,274 & $\mathbf{3 9 , 1}$ & 24,4 & 18,7 & 10,2 & 7,6 & 225 \\
\hline Páginas web & 3,47 & 1,035 & 4,9 & 10,7 & 32,4 & $\mathbf{3 6 , 4}$ & 15,6 & 225 \\
\hline Redes Sociales & 4,24 & 0,96 & 1,3 & 4,4 & 15,6 & 26,7 & $\mathbf{5 2}$ & 225 \\
\hline
\end{tabular}

Fuente: elaboración propia.

En esta primera tabla resulta muy interesante observar en los estadísticos básicos cómo las medias son realmente bajas en el caso de radio receptor $(M=1,71)$, radio online $(M=1,61)$ y periódicos $(M=1,7)$ correspondientes a valores de 'Nunca' muy altos: 53,3\%, 59,1\% y 52,4\% respectivamente; $\mathrm{y}$, por el contrario, cómo se muestra en aumento progresivo con la Prensa digital $(M=3,33)$, la TV $(M=3,45)$, TV online $(M=2,23)$ correspondiéndose a valores intermedios. Especialmente relevante es la media 
de uso de las redes sociales $(M=4,24)$ con un $52 \%$ de los encuestados contestando que 'Siempre' se informan por este medio.

Para una representación gráfica de estos valores se procede a reagrupar los valores extremos obviando los valores centrales, de modo que en la figura 2 se han agrupado las respuestas 'Nunca y Poco', por un lado, y luego las respuestas 'Con frecuencia y Siempre' por otro lado. El resultado en la figura 2 representa curvas con tendencias opuestas que se cruzan, mostrando que esta generación consume menos medios tradicionales y más medios digitales con una incidencia muy alta en el caso de la televisión (52,2\%), las páginas web $(52 \%)$ y las redes sociales $(78,7 \%)$.

Figura 2. Representación gráfica de los usos de los medios con valores reagrupados

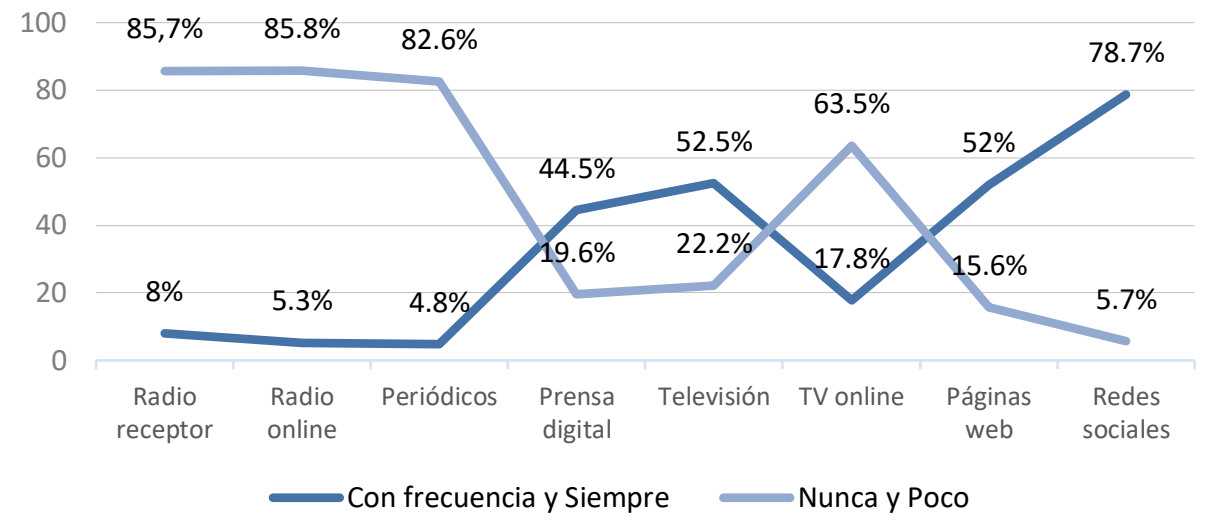

Fuente: elaboración propia.

A continuación, analizamos los descriptivos básicos para la $\mathrm{V}_{2}=$ Grado de fiabilidad que otorgas a esas fuentes, como se observa en la tabla 3.

Tabla 3. Análisis de la media, la desviación típica y la frecuencia para el estudio del grado de fiabilidad que otorgas a las fuentes por las que te informas

\begin{tabular}{|l|r|r|r|r|r|r|r|r|}
\hline $\begin{array}{l}\text { V2: ¿Qué grado } \\
\text { de fiabilidad le } \\
\text { otorgas a ... }\end{array}$ & Media & \multicolumn{1}{|c|}{ D.T. } & $\begin{array}{c}\text { Nunca } \\
(\mathbf{\%})\end{array}$ & $\begin{array}{c}\text { Poco } \\
(\%)\end{array}$ & $\begin{array}{c}\text { Ocasion } \\
\text { almente } \\
(\%)\end{array}$ & $\begin{array}{c}\text { Con } \\
\text { frecuenc } \\
\text { ia (\%) }\end{array}$ & $\begin{array}{c}\text { Siempr } \\
\text { e (\%) }\end{array}$ & N \\
\hline Radio receptor & 3,49 & 0,83 & 0,9 & 12 & 31,6 & $\mathbf{4 8 , 4}$ & 7,1 & 225 \\
\hline Radio online & 3,27 & 0,792 & 1,3 & 14,7 & 42,2 & $\mathbf{3 9 , 1}$ & 2,7 & 225 \\
\hline Periódicos & 3,56 & 0,89 & 1,8 & 10,2 & 29,3 & $\mathbf{4 7 , 1}$ & 11,6 & 225 \\
\hline Prensa digital & 3,27 & 0,872 & 1,3 & 16,9 & $\mathbf{4 2 , 2}$ & 32,4 & 7,1 & 225 \\
\hline Televisión & 3,26 & 0,981 & 3,6 & 20,4 & 29,3 & $\mathbf{3 9 , 6}$ & 7,1 & 225 \\
\hline TV online & 3,11 & 0,934 & 3,6 & 23,1 & $\mathbf{3 7 , 3}$ & 31,1 & 4,9 & 225 \\
\hline
\end{tabular}




\begin{tabular}{|l|r|r|r|r|r|r|r|r|}
\hline Páginas web & 2,64 & 0,828 & 5,3 & $\mathbf{4 0 , 9}$ & 39,1 & 13,3 & 1,3 & 225 \\
\hline Redes Sociales & 2,34 & 0,808 & 13,3 & $\mathbf{4 7 , 1}$ & 32,4 & 6,7 & 0,4 & 225 \\
\hline
\end{tabular}

Fuente: elaboración propia.

Los estadísticos básicos revelan que los encuestados otorgan una fiabilidad inversa a los medios relacionada con su consumo, es decir, que aquellos medios que más consumen - como se ha estudiado en los ítems anteriores- son a los que menos fiabilidad les otorgan y viceversa. Así, más de la mitad de los encuestados, el 55,5\%, otorga una fiabilidad muy alta a la radio (receptor), ocurre lo mismo con la radio online con un 41,8\%, y el 46,7\% confía mucho en la televisión (porcentajes resultado de sumar las respuestas de las respuestas 'Con frecuencia y Siempre'). Resultan ciertamente curiosos los datos de fiabilidad para los medios que más consumen, las páginas web con un 14,4\% de la muestra en confiabilidad alta y las redes sociales con un $7,1 \%$, teniendo el valor más bajo de todos.

\subsection{Consumo de redes sociales y rol asumido}

Respecto a este bloque, el análisis de los resultados responde a las preguntas

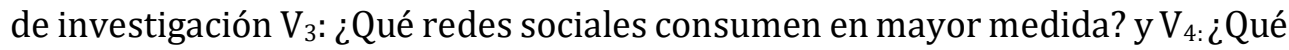
rol adoptan en su interacción en redes? Para la respuesta de la $V_{3}$ se utilizaron 7 ítems específicos de carácter cualitativo, categórico y ordinal con respuesta tipo Likert donde $1=$ Nada, $2=$ Algo, $3=$ Bastante, $4=$ Mucho y $5=$ Constantemente. En la tabla 4 observamos los resultados obtenidos.

Tabla 4. Análisis de la media, la desviación típica y la frecuencia para el estudio del grado de uso de las redes sociales

\begin{tabular}{|l|r|r|r|r|r|r|r|r|}
\hline $\begin{array}{l}\text { V.: cEn qué grado } \\
\text { usas las } \\
\text { siguientes redes } \\
\text { sociales? }\end{array}$ & Media & D.T. & $\begin{array}{c}\text { Nunca } \\
(\%)\end{array}$ & $\begin{array}{c}\text { Poco } \\
(\%)\end{array}$ & $\begin{array}{c}\text { Ocasion } \\
\text { almente } \\
(\%)\end{array}$ & $\begin{array}{r}\text { Con } \\
\text { frecuenc } \\
\text { ia (\%) }\end{array}$ & $\begin{array}{c}\text { Siempr } \\
\text { ( (\%) }\end{array}$ & N \\
\hline Facebook & 1,32 & 0,629 & $\mathbf{7 5 , 6}$ & 19,1 & 3,6 & 1,8 & 0 & 225 \\
\hline Instagram & 4,16 & 0,993 & 1,8 & 5,8 & 14,2 & 30,7 & $\mathbf{4 7 , 6}$ & 225 \\
\hline Tik Tok & 2,36 & 1,401 & $\mathbf{4 0}$ & 20,4 & 13,8 & 15,6 & 10,2 & 225 \\
\hline Twitter & 3,02 & 1,479 & 21,3 & 20,9 & 15,6 & 19,1 & $\mathbf{2 3 , 1}$ & 225 \\
\hline Whatsapp & 4,24 & 0,99 & 0 & 8,4 & 14,2 & 21,8 & $\mathbf{5 5 , 6}$ & 225 \\
\hline Twitch & 1,44 & 0,885 & $\mathbf{7 4 , 7}$ & 12,9 & 8 & 2,7 & 1,8 & 225 \\
\hline YouTube & 3,39 & 1,081 & 1,3 & 23,1 & $\mathbf{2 9 , 8}$ & $\mathbf{2 6 , 7}$ & 19,1 & 225 \\
\hline
\end{tabular}

El análisis descriptivo da una idea clara de las redes sociales utilizadas por la generación Z: en el caso Facebook encontramos un 75,6\% de los encuestados que nunca utiliza esta red; en el caso de Instagram, un 78,3\% la 
utiliza con frecuencia o siempre; para el uso de TikTok también encontramos un porcentaje ato de personas $(60,4 \%)$ que respondieron no usarla nunca o poco; la red Twitter encuentra $(M=3,02$; $D T=1,479)$ una distribución de respuestas bastante igualada, lo que indica un uso intermedio, con porcentaje parecido entre los que nunca lo utilizan $(21,3 \%)$ y los que declaran utilizarlo siempre $(23,1 \%)$; respecto a WhatsApp $(M=4,24$; $D T=0,99)$, su uso resulta imbatible, el 77,4\% de los encuestados declara usarlo con frecuencia o siempre, y no hay nadie en la muestra que no lo use. Los datos para Twitch entre los encuestados muestran un $74,7 \%$ que afirma no usarlo nunca y, en el caso de YouTube, ocurre parecido a WhatsApp: prácticamente todos lo utilizan, el dato más alto es su uso ocasional por un tercio de la muestra $(29,8 \%)$.

Analizando los datos de modo gráfico en la figura 3, se observa que las dos redes sociales más usadas 'Constantemente' son WhatsApp e Instagram, con $55,6 \%$ y $47,6 \%$ respectivamente; mientras que las menos usadas son Twitch y Facebook con 74,7\% y 75,6\% que declaran no usarlas 'Nunca'.

Figura 3. Uso de redes sociales en porcentajes

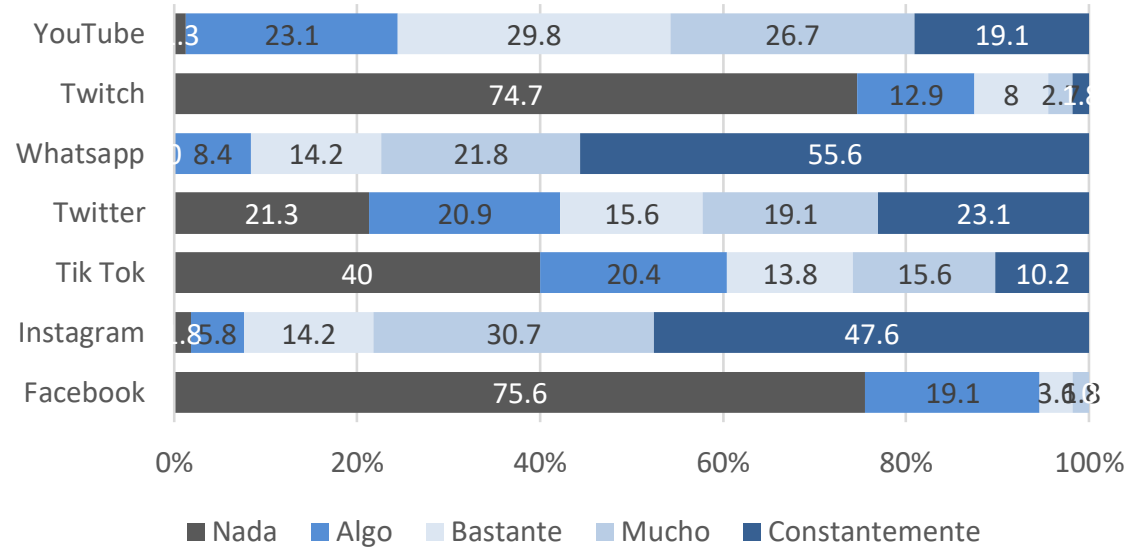

Fuente: elaboración propia.

Con la intención de profundizar en el tipo de interacción que los usuarios mantienen en redes, en la muestra objeto de estudio, se les pregunta además por su rol al utilizarlas. Esta variable $\left(\mathrm{V}_{4}\right)$ de tipo cualitativo, categórica y ordinal se codificó con cinco tipos de respuesta en las que se categorizó el nivel de engagement del usuario en su interacción, siendo 1 el de menor interacción y 5 el de máximo grado: Grado 1 = «Solo consumo sin participar»; Grado $2=$ «Consumo y participo compartiendo contenido»; Grado $3=$ "Consumo, participo compartiendo y comento»; Grado $4=$ «Consumo, participo 
compartiendo, comento y busco mencionar a otros» y Grado $5=$ «Consumo, participo compartiendo, comento y busco controversia/disputa».

Figura 4. Grados de participación en redes (en porcentajes)

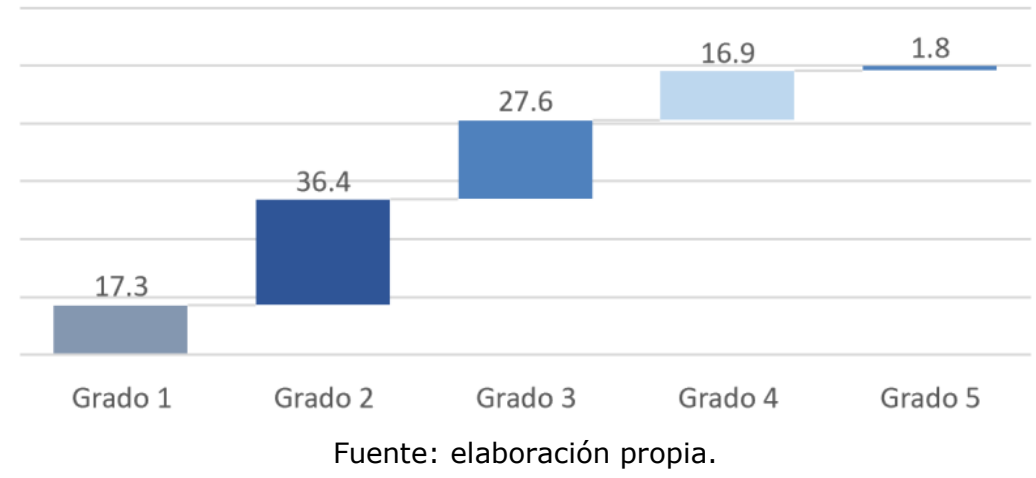

\subsection{Distinción de fake news y su asociación a las redes sociales}

Por último, en este tercer bloque, el análisis de los resultados responde a las preguntas de investigación relacionadas con las fake news, $\mathrm{V}_{5}$ : Son capaces de distinguir fake news (figura 5) y $\mathrm{V}_{6}$ : Qué redes sociales se asocian más a la distribución de fake news. Es importante destacar que los resultados de este bloque han sido fruto de la recogida de las percepciones de la muestra, por lo que su interpretación debe hacerse teniendo en cuenta esta consideración.

Figura 5. Porcentaje de los encuestado que distingue fake news en su consumo de medios

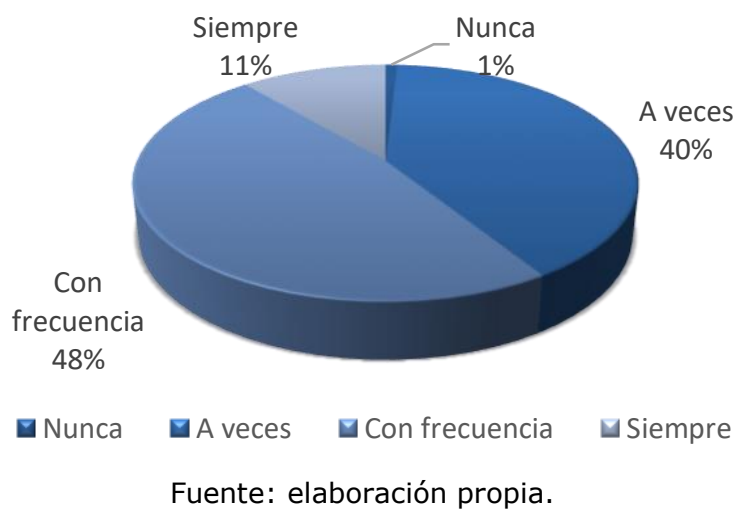


Como podemos observar en la figura 5, prácticamente todos los encuestados distinguen con diferente grado de frecuencia las noticias falsas cuando consumen medios del tipo que sea. Un 11\% declara que siempre las distingue, prácticamente la mitad (un 48\%) afirma que las distingue con frecuencia y un $40 \%$ sólo las distingue a veces.

Respecto a la asociación que se hace de la distribución de las fake news con respecto a los medios, los resultados nos indican, como puede verse en la tabla 5, cuáles son las percepciones de los encuestados.

Tabla 5. Análisis de la media, la desviación típica y la frecuencia para el estudio del grado de uso de las redes sociales

\begin{tabular}{|l|r|r|r|r|r|r|r|r|}
\hline $\begin{array}{l}\mathbf{V}_{6}: \text { ¿A qué medios } \\
\text { asocias más la } \\
\text { distribución de } \\
\text { fake? }\end{array}$ & Media & D.T. & $\begin{array}{c}\text { Nunca } \\
(\%)\end{array}$ & $\begin{array}{c}\text { Poco } \\
(\%)\end{array}$ & $\begin{array}{c}\text { Ocasion } \\
\text { almente } \\
(\%)\end{array}$ & $\begin{array}{c}\text { Con } \\
\text { frecuenc } \\
\text { ia (\%) }\end{array}$ & $\begin{array}{c}\text { Siemp } \\
\text { re (\%) }\end{array}$ & N \\
\hline $\begin{array}{l}\text { A medios } \\
\text { tradicionales }\end{array}$ & 2,75 & 1,068 & 8 & 41,1 & 26,8 & 16,5 & 7,6 & 225 \\
\hline A páginas web & 3,68 & 0,839 & 1,3 & 6,7 & 28,1 & $\mathbf{5 0 , 4}$ & 13,4 & 225 \\
\hline A Twitter & 3,87 & 0,79 & 4,9 & 23,7 & 50,9 & 20,5 & 11,6 & 225 \\
\hline A Instagram & 3,74 & 0,835 & 8 & 27,2 & 47,8 & 17 & 7,1 & 225 \\
\hline A Facebook & 3,88 & 0,864 & 0,4 & 5,4 & 25,4 & 43,8 & 25 & 225 \\
\hline A TikTok & 3,33 & 1,186 & 8,9 & 16,1 & 24,1 & 34,8 & 16,1 & 225 \\
\hline A Whatsapp & 3,97 & 0,944 & 0,9 & 7,6 & 17,9 & 41,1 & $\mathbf{3 2 , 6}$ & 225 \\
\hline Al boca a boca & 4 & 0,925 & 0,4 & 7,6 & 17,4 & 41,1 & 33,5 & 225 \\
\hline
\end{tabular}

Fuente: elaboración propia.

De estos resultados cabe destacar una clara tendencia de la generación $\mathrm{Z}$ a asociar la distribución de las fake news con todos los canales digitales, el $50,9 \%$ percibe que en Instagram se distribuyen noticias falsas 'Con frecuencia y Siempre', el 68,8\% asocia su distribución en las páginas web, el 68,8\% lo asocian a Facebook, sólo superado por el 73,7\% que asocian la distribución a través de Whatsapp y el 74,6\%, que lo asocia al boca a boca. Valores bastante intermedios encontramos en el caso de Twitter, un tercio de la muestra solamente asocia la red a distribución de fakes, y en el caso de Instagram sólo un $24,1 \%$, un valor idéntico al asociado a los medios tradicionales. 


\section{Figura 6. Representación gráfica de la percepción en la distribución} de fake news por diferentes canales

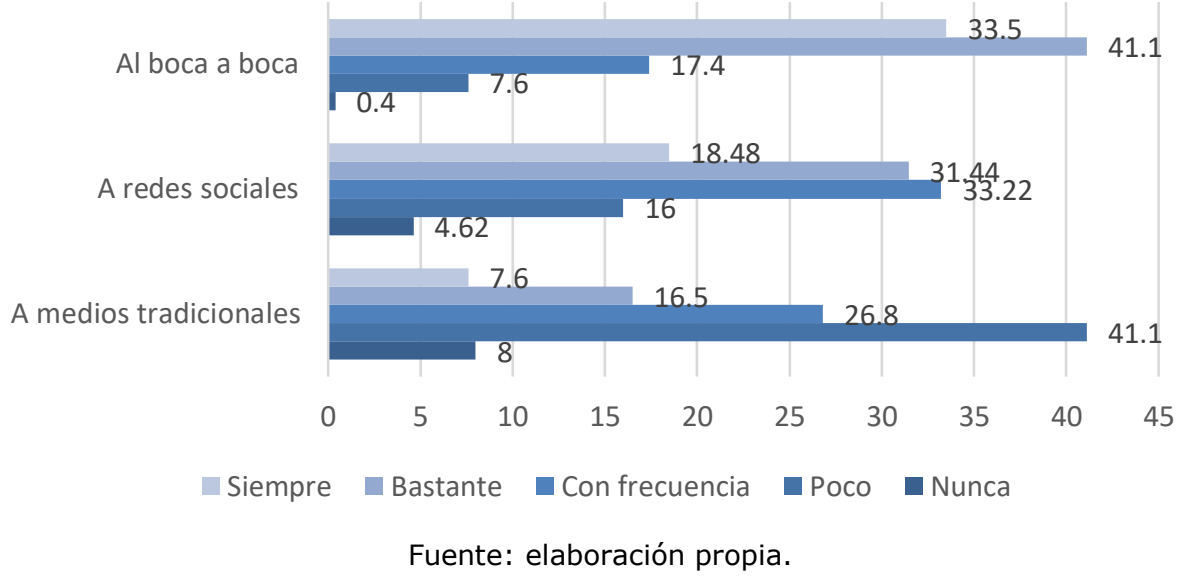

Si reagrupamos los datos en medios tradicionales, redes sociales y boca a boca, el resultado gráfico lo tenemos en la figura 6, en la que se observa que según la muestra de estudio los medios tradicionales serían los mejor considerados en la distribución de fake news; prácticamente la mitad de la muestra $(49,1 \%)$ considera que distribuyen poco o nada este tipo de contenidos, un $26,8 \%$ considera que con frecuencia y sólo un 7,6\% declara que siempre. Respecto a los otros dos medios de distribución agrupados, las redes sociales suponen el medio que más se asocia con la distribución de fake news, el $49,92 \%$ considera que siempre o bastante y el 33,22\% contesta que con frecuencia. Sin embargo, los datos más altos los encontramos en el boca a boca, donde el $74,6 \%$ de los encuestados considera que las noticias falsas se distribuyen 'Siempre' y 'Bastante'.

\section{Discusión y conclusiones}

La transformación digital ocurrida en las últimas décadas, acelerada sin duda por una crisis global debida a la pandemia por Covid-19 y amplificada por las redes sociales, deja un panorama de consumo totalmente distinto y decisivo no sólo para los medios, sino para las plataformas digitales que se erigen como acompañantes sempiternos de una población siempre pendiente de su móvil (Gómez-Calderón et al., 2020; Lenhart et al., 2010; Pérez-Escoda et al., 2021). A los estudios previos que han dado cuenta de la distancia cada vez mayor entre las audiencias jóvenes y los medios como la televisión (García-Avilés, 2020), la radio (Pérez-Maíllo et al., 2018; Pedrero-Esteban, 2014) o la prensa (Yuste, 2020), se 
suman circunstancias que no mejoran esta situación. Una sociedad ampliamente mediatizada (Pérez-Tornero, 2020) y una pandemia reciente han dejado evidencias claras de una desconexión absoluta entre los públicos jóvenes y los medios (Tayal y Bharathi, 2021; Vintimilla y Torres-Toukumidis, 2021).

El estudio que aquí se presenta aporta como novedad resultados preliminares de una generación que consume los medios en los que menos confía, lo cual supone una paradoja interesante desde el punto de vista de la educación en medios que se impone más que nunca (Castillo-Abdul, RomeroRodríguez y Larrea-Ayala, 2020; Pérez-Escoda et al., 2021). Sin embargo, cabe destacar una diferencia importante con respecto a estudios anteriores que ponían el acento en la necesidad de una alfabetización mediática de uso y acceso (García-Ruiz et al., 2018) es interesante subrayar que lo que se detecta en este estudio es la necesidad de un enfoque más competencial, crítico y responsable (García-Ruiz y Pérez-Escoda, 2021).

No obstante, es importante mencionar que una de las limitaciones del estudio es el tamaño de la muestra, que resulta reducido, de ahí que el estudio no pretenda hacer inferencias, sino describir de modo general ciertas tendencias que podrían ser confirmadas en estudios más amplios. Aunque los resultados están alineados con otros estudios académicos recientes (CatalinaGarcía, Sousa y Silva, 2019; Patch, 2018; Rubio-Romero y Barón-Dulce, 2019; Sádaba y Pérez-Escoda, 2020), también con el último Estudio de Redes Sociales en España (IAB, 2020), resulta interesante destacar la incoherencia en el consumo de medios que se trasluce en los resultados. La generación $\mathrm{Z}$ consume más aquellos medios que menor fiabilidad le merecen y aunque los relacionan con la distribución de fake news son los que más consultan para estar informados; todo ello teniendo en cuenta que casi la mitad afirma que sólo a veces distinguen las fake news, lo que confirma una necesidad formativa al respecto. En contraposición con otros trabajos (Gómez Calderón, CórdobaCabús y Méndez-Nieto, 2020), los medios de comunicación tradicionales son identificados como más fiables que las redes sociales, aunque resulta interesante que coincide con esos trabajos en que los entornos cercanos, de boca a boca, resultan identificados por esta generación como los más propicios para la distribución de fake news.

La reflexión final que surge a partir de estudios de este corte es que estamos ante una generación intensamente expuesta a medios digitales que ellos mismos reconocen como poco fiables, pero que son a los que están conectados (Álvarez-Ramos, Heredia-Ponce y Romero-Olive, 2019). Según el estudio publicado por la Organización Mundial de Trabajadores (OIT, 2020), realizado a 12.000 jóvenes de 112 países, la pandemia ha acentuado hábitos y 
exposiciones que claramente abocan a una desinformación y efectos «cámara de eco» o «burbuja de filtros». Lo más preocupante ante esta situación es la percepción y actitud de la población más joven ante el fenómeno de las noticias falsas que va cambiando de una actitud más proactiva hace unos años, como lo muestra el trabajo de Catalina-García et al. $(2017,2019)$, hacia actitudes más pasivas en las que no sólo no existe verificación o fact-checking, sino que declaran un uso intensivo de aquellos medios que a su vez reconocen como más propensos a contener y difundir noticias falsas. Estas evidencias hacen necesarias acciones de formación y alfabetización que ayuden a las generaciones más jóvenes (aunque también a toda la ciudadanía en general) a reaccionar ante un bucle de consumo mediático perjudicial globalmente mediatizado (Pérez-Tornero, 2020).

\section{Referencias bibliográficas}

ALLCOTT, H. \& GENTZKOW, M. (2017). Social media and fake news in the 2016 election. Journal of Economic Perspectives, 31(2), 211-236. doi.org/10.1257/jep.31.2.211

Álvarez RAmOS, E.; HEREDIA PonCE, H. y Romero Olive, M. (2019). La generación $\mathrm{Z}$ y las redes sociales. Una visión desde los adolescentes en España. Revista Espacios, 40(20), 1-13. Disponible en: https: / / tinyurl.com/v9fc5hd2

BARÓN-DULCE, G. (2017). Una aproximación a la relación de los jóvenes con las marcas. Las redes sociales como escenario. Tesis doctoral, Universidad Nebrija, Madrid. Disponible en: https: //tinyurl.com/4e5pvwuf

Carrera, P.; Blanco-Ruiz, M. y Sainz de Baranda-AndújAR, C. (2020). Consumo mediático entre adolescentes. Nuevos medios y viejos relatos en el entorno transmedia. Historia y Comunicación Social, 25 (2), 563-574.

\section{doi.org/10.5209/hics.72285}

CASTILLO-ABDUL, B.; ROMERO-RodRíGUEZ, L.M.J. y LARREA-AYALA, A. (2020). Kid influencers in Spain: understanding the themes they address and preteens' engagement with their YouTube channels. Heliyon, 6(9), doi.org/10.1016/j.heliyon.2020.e05056

CATAlinA-GARCíA, B.; VozMEdiano, M.M. y GARCía-JimÉnEZ, A. (2017). Los jóvenes universitarios y sus pautas de consumo y difusión de noticias según la tendencia ideológica. Fonseca, Journal of Communication, 15, 57-73. doi.org/10.14201/fjc2017155773

Catalina-GarCía, B.; SousA, J.P. y CRISTINA-SILVA, L.C. (2019). Consumo de noticias y percepción de fake news entre estudiantes de Comunicación de Brasil, España y Portugal. Revista de Comunicación, 18(2), 93-115.

doi.org/10.26441/RC18.2-2019-A5 
Creswell, J.W., \& Poth, C.N. (2016). Qualitative inquiry \& research design: Choosing among five approaches. (4th ed). SAGE Publications.

COMISIÓN EUROPEA (2018). Representación en España. Contra las noticias falsas: el Grupo de Expertos pide transparencia a las plataformas en línea. Disponible en https: / / tinyurl.com/57dzdmas

COMISIÓN EUROPEA (2019). Eurobarómetro Standard 92. Informe Nacional:

España. Comisión Europea. Disponible en: https://tinyurl.com/cza9kfnn ESPIRITUSANTO, O. (2016). Los auténticos nativos digitales: ¿Estamos preparados para la Generación Z? Revista de Estudios de Juventud, IN-JUVE, no14, 111-126. Disponible en: https: / / tinyurl.com/243rr23j

GARCÍA-AvILÉS, J.A. (2020). Reinventing Television News: Innovative Formats in a Social Media Environment. In J. Vázquez-Herrero, S. Direito-Rebollal, A. Silva-Rodríguez and X. López-García (Ed.). Journalistic Metamorphosis. Cham: Springer, pp. 143-55. Disponible en:

https: //tinyurl.com/7f3575xn

GARCÍA-RuIZ, R.; RodríGuEZ, A. y TORRES, A. (EDS.) (2018). Educar para los nuevos medios: Claves para el desarrollo de la competencia mediática en el entorno digital. Ecuador: Abya Yala.

GARcíA-RUIZ, R. y PÉREZ-EscodA, A. (2021). La competencia digital como clave para fortalecer el uso responsable de internet. Campus Virtuales, 10, 59-71. Disponible en: https: / /tinyurl.com/2wudz7r9

Gómez CALDERón, B.; CóRdobA-CABúS, A. y MÉndez NiETo, A. (2020). Jóvenes y fake news. Un análisis sociodemográfico aplicado al caso andaluz. Revista Científica de Información y Comunicación, 17, 481-501. Disponible en: https: / /tinyurl.com/3nj4tu38

GonZÁLEZ AlONSO, J. y PAZMiÑo SANTACRUZ, M. (2015). Cálculo e interpretación del Alfa de Cronbach para el caso de validación de la consistencia interna de un cuestionario, con dos posibles escalas tipo Likert. Revista Publicando, 2(1), 62-67. Recuperado de: https: / / tinyurl.com/yuu4cm67 HERrero-Diz, P.; CONDE-JIMÉNEZ J. y REYES DE CóZAR S. (2020). Teens' Motivations to Spread Fake News on WhatsApp. Social Media + Society. July 2020. doi: 10.1177/2056305120942879

IAB (2020). Estudio de Redes Sociales 2020. Disponible en:

https: / / tinyurl.com/2p34rkxc

LENHART, A.; PURCEL, K.; SMith, A. y ZiCKuHr, K. (2010). Social Media and movil Internet use among teens and young adults. Pew Internet and American life. Disponible en: https: / / tinyurl.com/29na872a 
LEVY, D.; NEWMAN, N.; FletCheR, R.; KALOGEROPOUlOS, A. \& NiELSEN, R. K. (2019). Reuters Institute Digital News Report 2019. Report of the Reuters Institute for the Study of Journalism. Disponible en: https: //tinyurl.com/89w28n74

Mendiguren, T.; PÉrez Dasilva, J. y Meso Ayerdi, K. (2020). Actitud ante las Fake News: Estudio del caso de los estudiantes de la Universidad del País Vasco. Revista de Comunicación, 19(1), 171-184. doi.org/10.26441/RC19.1-2020-A10

Negredo, S.; AMOEdo, A.; VARA Miguel, A.; Moreno, E. \& KAUfmanN, J. (2020). Digital News Report.es 2020. Disponible en: https://tinyurl.com/x6c6mzwd

NeWMAN, N.; FleTCHER, R.; KAlOGEROPOUlOS, A.; LEVY, D. \& NiELSEN, R.K. (2017). Reuters Institute Digital News Report 2017. Disponible en: https: //tinyurl.com/hccrvcjc

OIT (2020). Los jóvenes y la COVID-19: Efectos en los empleos, la educación, los derechos y el bienestar mental. Disponible en: https: / / tinyurl.com / 2c2mjbxv

PATCH, H. (2018). Which Factors Influence Generation Z's Content Selection in OTT TV?: A Case Study. Tesis doctoral. School of Electrical Engineering and Computer Science. Disponible en: https://tinyurl.com/35n7m9mj

PEDRERo-EstebAN, L.M. (2014). Main challenges of Spanish musicradio in the age of Spotify. En F. Ubierna y J. Sierra (Coord.). Miscelánea sobre el entorno audiovisual en 2014. (pp. 441-456). España: Fragua.

PÉREZ-EsCodA, A.; LENA-ACEBO, F.J. y GARCÍA-RUIZ, R. (2021). Brecha digital de género y competencia digital entre estudiantes universitarios. Aula Abierta, 50, 505-514. doi.org/10.17811/rifie.50.1.2021.505-5014

PÉrez-Escoda, A.; PEdrero-Esteban, L.M.; Rubio-Romero, J. \& JimÉnEZ-NARRos, C. (2021). Fake news reaching young people on social networks: Distrust challenging media literacy. Publications, 9(2), 24. doi.org/10.3390/publications9020024

PÉreZ-Maíllo, A.; SÁnCHEZ-SERRANo, C. y PEDRERo-EstebAN, L.M. (2018). Viaje al Centro de la Radio. Diseño de una experiencia de alfabetización transmedia para promover la cultura radiofónica entre los jóvenes. Comunicación y Sociedad, 33, 171-201. doi.org/10.32870/cys.v0i33.7031

PÉREZ-ToRnERo, J.M. (2020). La gran mediatización. Barcelona, España: UOC Editorial.

QUANTILOPE INC. (2021). What do Consumers Expect from Brands in 2021 Around Diversity \& Inclusion? Insights 2021. Disponible en: https: / / tinyurl.com/4dad9w9k 
RODRÍGUEZ-FERNÁNDEZ, L. (2019). Desinformación: retos profesionales para el sector de la comunicación. Profesional de la Información, 28(3). doi.org/10.3145/epi.2019.may.06

Rubio-Romero, J. y BARÓN-DulCE, G. (2019). Actitudes de los jóvenes hacia las comunidades virtuales y su vínculo con las marcas. Una aproximación a través de los estudiantes universitarios de comunicación y de marketing de la Universidad Nebrija. AdComunica, 18, 41-62. doi.org/10.6035/2174-0992.2019.18.4

SÁDABA-CHALEZQUER, C. y PÉREZ-ESCODA, A. (2020). La generación «streaming» y el nuevo paradigma de la comunicación digital. En L.M. Pedrero-Esteban y A. Pérez-Escoda, Cartografía de la comunicación postdigital: Medios y audiencias en la Sociedad de la COVID-19. Navarra, España: CivitasThomson Reuters. (pp. 97-114).

Schroer, W. (2008). Generations X, Y, Z and the others. The Journal of the Household Goods Forwarders Association of America, Inc, 40, 9-11.

Disponible en: https: / /tinyurl.com/b8xcd67s

TANDOC JR. E.; LIM, Z. \& LING, R. (2018) Defining “Fake News”. Digital Journalism, 6:2, 137-153, doi.org/10.1080/21670811.2017.1360143

TAYAL, P. \& BHARATHI. V. (2021) Reliability and trust perception of users on social media posts related to the ongoing COVID-19 pandemic. Journal of Human Behavior in the Social Environment, 31:1-4, 325-339, doi.org/10.1080/10911359.2020.1825254

VALERO, P.P. \& OLIVEIRA, L. (2018). Fake news: una revisión sistemática de la literatura. Observatorio $\left(O B S^{*}\right), 12(5), 54-78$. doi.org/10.15847/obsOBS12520181374

VinTimilla-LEÓn, D. y ToRRES-TOUKOUMIDIS, A. (2021). COVID-19 y Tik-Tok. Análisis de la Folksonomía social. RISTI, Revista Ibérica de Sistemas e Tecnologías de la Informaçao, 39(5), 15-26. Disponible en: https: / / tinyurl.com/che3fefm

WE ARE Social (2021). Digital Report 2021. El informe de las tendencias digitales. Disponible en: https: / / tinyurl.com/uectedjd

YUSTE, B. (2020). Las nuevas formas de consumir información de los jóvenes. Revista de estudios de juventud. INJUVE, 108 (15). Disponible en https: / /tinyurl.com/ymtwfje8 